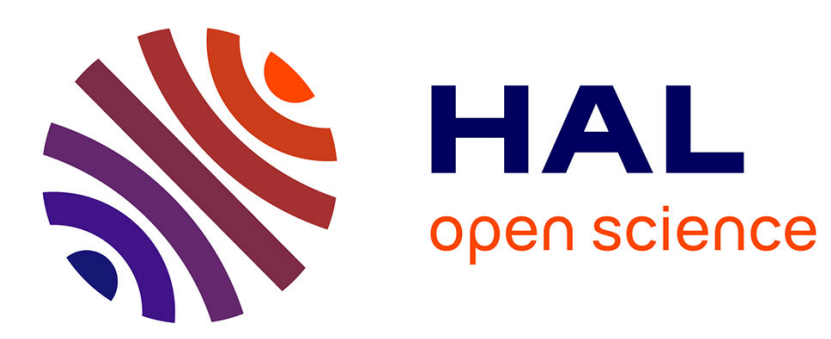

\title{
Stochastic finite differences for elliptic diffusion equations in stratified domains
}

\author{
Sylvain Maire, Giang Nguyen
}

\section{To cite this version:}

Sylvain Maire, Giang Nguyen. Stochastic finite differences for elliptic diffusion equations in stratified domains. Mathematics and Computers in Simulation, 2016, 121, 10.1016/j.matcom.2015.09.008 . hal-00809203v2

\section{HAL Id: hal-00809203 https://inria.hal.science/hal-00809203v2}

Submitted on 19 Sep 2013

HAL is a multi-disciplinary open access archive for the deposit and dissemination of scientific research documents, whether they are published or not. The documents may come from teaching and research institutions in France or abroad, or from public or private research centers.
L'archive ouverte pluridisciplinaire HAL, est destinée au dépôt et à la diffusion de documents scientifiques de niveau recherche, publiés ou non, émanant des établissements d'enseignement et de recherche français ou étrangers, des laboratoires publics ou privés. 


\title{
Stochastic finite differences for elliptic diffusion equations in stratified domains
}

\author{
Sylvain Maire Giang Nguyen *
}

September 17, 2013

\begin{abstract}
We describe Monte Carlo algorithms to solve elliptic partial differential equations with piecewise constant diffusion coefficients and general boundary conditions including Robin and transmission conditions as well as a damping term. The treatment of the boundary conditions is done via stochastic finite differences techniques which possess an higher order than the usual methods. The simulation of Brownian paths inside the domain relies on variations around the walk on spheres method with or without killing. We check numerically the efficiency of our algorithms on various examples of diffusion equations illustrating each of the new techniques introduced here.
\end{abstract}

Keywords: Monte Carlo method, Elliptic diffusion equations, Stratified media, Stochastic finite differences, Walk on spheres.

\section{Introduction}

Many physical models assume that the flow is proportional to the gradient of the concentration of an incompressible fluid in an homogeneous media. In such models, the concentration is hence the solution of an equation involving the Laplace operator. In more realistic physical situations, the diffusivity coefficient is only piecewise constant so that the laplace operator needs to be replaced by an operator that takes a divergence form. Such situations occur for instance in geophysics [12], magneto-electro-encephalography [19], population ecology [4] or astrophysics [29]. Solving the resulting partial differential equations is quite challenging as they hold in large domains presenting complex geometries and multi-scale features. Another very important issue is the resolution of inverse problems which occur in electrical impedance tomography [10] for example for the detection of breast cancer. The numerical resolution of

\footnotetext{
${ }^{*}$ Université de Toulon, CNRS, LSIS, UMR 729683957 La Garde, France. Email: [maire, tnguyen] @univ-tln.fr
} 
these inverse problems is most of the time coupled with an iterative method. This involves many resolutions of forward problems which makes the numerical resolution of these forward problems even more crucial.

Obviously very efficient deterministic or probabilistic methods exist to solve problems involving the Laplace operator. Monte Carlo algorithms rely on the simulation of the Brownian motion using random walks on subdomains methods like the walk on spheres (WOS) [24] or more recently the walk on rectangles (WOR) [5]. The crucial point when dealing with discontinuous media is the behavior of the random walk when it hits the interface between physical subdomains. Many algorithms essentially monodimensional have been proposed to deal with this interface conditions $[7,12,19]$. In recent works, some new approaches have been introduced based either on stochastic processes tools $[14,15,25]$ or on finite differences techniques $[2,20]$. In particular, both methodologies have been improved and compared in [16] on elliptic, parabolic and eigenvalue problems.

Another important issue is the treatment of boundary conditions like Dirichlet, Neumann or more generally Robin boundary conditions. The simulation of general diffusions via the Euler scheme in presence of respectively Dirichlet and Neumann boundary conditions have been studied for instance in [8] and $[3,17]$ respectively. Robin boundary conditions are treated in [21] but for a walk on a fixed cartesian grid. The layer method introduced in [22] enables to deal with these Robin conditions for parabolic problems and very general diffusion operators. A discussion between Robin boundary conditions and the simulation of diffusion processes is done in [6]. The exit time of subdiffusions in a bounded domain with homogeneous Robin conditions is studied in [9] using spectral theory. The WOS deals very naturally and efficiently with Dirichlet boundary conditions [24] and it can be coupled with stochastic finite differences to deal with Neumann boundary conditions [17].

The aim of this paper is to provide efficient Monte Carlo methods to deal with elliptic partial differential equations in dimensions two and three with a piecewise constant diffusion coefficient, Robin boundary conditions and also a linear damping term in the equation. The treatment of boundary conditions relies on high order stochastic finite differences generalizing the methodology developed in [16] and [17] to more complex equations and to higher dimensions while the interior simulation of the Brownian paths is mainly based on the WOS method. We focus on the quality of the schemes that take the transmission and the boundary conditions into account. Nevertheless, we also pay attention on the walk on spheres dynamics and scoring especially in the presence of both a damping term and a source term.

The rest of the paper is organized as follows. In section 2 , we remind the Feynman-Kac formula which give the probabilistic representations of the solution of the elliptic diffusion equations. We also explain the general algorithm where we compute a score along a random walk until its killing due to the damping term or to its absorption by the boundary. This algorithm relies on two fundamental tools. The walk on spheres described in section 3 enables the 
simulation of the Brownian path and its relative score away from the boundary or the physical interfaces of the domain. The stochastic finite differences method introduced in section 4 deals with the dynamics and the scoring of the path when it reaches a physical interface or the boundary of the domain. In the final section, we give some numerical examples to illustrate the new schemes introduced before especially for Robin boundary conditions and for equations involving a damping term. In this last situation, we also make some comparisons between WOS simulations and simulations based on the Euler scheme.

\section{Feynman-Kac Formula and double random- ization}

We want to solve by means of a Monte Carlo method elliptic partial differential equations of the form

$$
\left\{\begin{array}{c}
-\frac{1}{2} \nabla(a(x) \nabla u(x))+\lambda(x) u(x)=f(x), \quad x \in D \\
\alpha(x) u(x)+\beta(x) \frac{\partial u(x)}{\partial n}=g(x), \quad x \in \partial D
\end{array}\right.
$$

in a domain $D$ divided in subdomains in which both the diffusion coefficient $a(x)>0$ and the damping coefficient $\lambda(x) \geqslant 0$ are constant. The positive coefficients $\alpha$ and $\beta$ (which cannot vanish simultaneously) may depend on $x$ which is often the case in real applications. For example in electrical impedance tomography applied to breast cancer, the tumors are modelized by Dirichlet conditions, the electrodes by Robin conditions and the rest of the skin by Neumann conditions. We assume that this equation has a unique solution which essentially means that we are not in the pure Neumann case that is $\alpha=0$ and $\lambda=0$ everywhere. Our Monte Carlo method is based on the evolution of a particle and of its score along a path that goes from one subdomain to another until it is killed due to the boundary conditions or to the damping term. It is constituted of two main steps: a walk inside each subdomain with Dirichlet boundary conditions and a replacement when hitting an interface between subdomains or the boundary of $D$. The validity of the algorithm is obtain thanks to the double randomization principal.

\subsection{Feynman-Kac formula for Poisson type equations}

The Feynman-Kac formula is a very powerful tool to write the pointwise solution of linear transport or diffusion equations as the mean value of a functional of a stochastic process [13]. This pointwise solution is computed numerically by means of a Monte Carlo method. Besides the usual Monte Carlo error, an additional bias may appear due to the approximations of this stochastic process by a discretization scheme. For general diffusion equations, this discretization is usually done via the Euler scheme with stepsize $\Delta t$. This induces a weak 
error of an order $O(\Delta t)$ for problems in the whole space [27]. For Dirichlet boundary conditions, the weak error using the naive Euler scheme is a $O(\sqrt{\Delta t})$ but it can be reduced to a $O(\Delta t)$ thanks to the half-space approximation [8].

Our algorithm requires to solve the Poisson equation with Dirichlet boundary conditions and an additional damping term in a domain $D \subset \mathbb{R}^{d}$ with a piecewise smooth boundary $\partial D$

$$
\left\{\begin{array}{c}
-\frac{1}{2} \Delta u(x)+\lambda u(x)=f(x), \quad x \in D \\
u(x)=g(x), \quad x \in \partial D
\end{array}\right.
$$

where $\lambda \geqslant 0$ is a constant damping parameter. The Feynman-Kac formula gives the stochastic representation of the solution by

$$
u(x)=\mathbb{E}_{x}\left[g\left(W_{\tau_{D}}\right) e^{-\lambda \tau_{D}}+\int_{0}^{\tau_{D}} f\left(W_{t}\right) e^{-\lambda t} d t\right]
$$

where $\left(W_{t}\right)_{t \geqslant 0}$ is a Brownian motion in dimension $d$ and $\tau_{D}$ its first exit time from $D$. For the Poisson equation more efficient techniques than the Euler scheme exist like the WOS or WOR which are especially designed for the simulation of Brownian paths. The WOR enables to treat more precisely parabolic or eigenvalue problems [16] than the WOS method. However we use the WOS method in all the following because it is somehow easier to handle and that we only deal with elliptic problems.

\subsection{Double randomization}

To solve the equation in the whole domain $D$, we need to solve equations in subdomains where the diffusion coefficient $a$ and the damping coefficient $\lambda$ are constant. Denoting by $a_{1}$ and $\lambda_{1}$ these two coefficients in a given subdomain $D_{1}$, the restriction $u_{1}$ to $D_{1}$ of the solution $u$ of 2.1 verifies

$$
\left\{\begin{array}{c}
-\frac{a_{1}}{2} \Delta u_{1}(x)+\lambda_{1} u_{1}(x)=f(x), \quad x \in D_{1} \\
u_{1}(x)=u(x), \quad x \in \partial D_{1} .
\end{array}\right.
$$

The stochastic representation of the solution is

$$
u_{1}(x)=\mathbb{E}_{x}\left[u\left(W_{\tau_{D}}\right) e^{-\frac{\lambda_{1}}{a_{1}} \tau_{D}}+\int_{0}^{\tau_{D}} \frac{1}{a_{1}} f\left(W_{t}\right) e^{-\frac{\lambda_{1}}{a_{1}} t} d t\right]
$$

where $\left(W_{t}\right)_{t \geqslant 0}$ is still a Brownian motion in dimension $d$. Obviously, $u(x)$ is not known for a point $x$ on the boundary of $D_{1}$ but we will develop in section 4 a method which express this quantity as the mean value of a random variable plus a bias due to a finite differences approximation. In the case of Robin boundary conditions, this method leads to

$$
u(x)=\sum_{i=1}^{N} p_{i} u_{1}\left(x_{i}\right)+c f(y)+d g(x)+O\left(h^{3}\right)
$$


where the coefficients $p_{i}>0$ verify $\sum_{i=1}^{N} p_{i} \leqslant 1$ and $h$ is the finite differences step. The $N$ points $x_{i}$ and the point $y$ are located in the subdomain $D_{1}$. When the Robin boundary is hitten by the motion, the quantity

$$
c f(y)+d g(x)
$$

is added to the score. The motion is killed with probability $1-\sum_{i=1}^{N} p_{i}$ and otherwise replaced according to the $p_{i}$ at one of the $x_{i}$. In the case of transmissions conditions between two subdomains $D_{1}$ and $D_{2}$, we have

$$
u(x)=\sum_{i=1}^{N} p_{i} u_{1}\left(x_{i}^{(1)}\right)+\sum_{i=1}^{N} q_{i} u_{2}\left(x_{i}^{(2)}\right)+c_{1} f\left(y_{1}\right)+c_{2} f\left(y_{2}\right)+O\left(h^{3}\right)
$$

where the coefficients $p_{i}>0$ and $q_{i}>0$ verify $\sum_{i=1}^{N}\left(p_{i}+q_{i}\right) \leqslant 1$ and $h$ is the finite differences step. When the interface is hitten by the motion, the quantity

$$
c_{1} f\left(y_{1}\right)+c_{2} f\left(y_{2}\right)
$$

is added to the score. The $N$ points $x_{i}^{(1)}$ and $y_{1}$ are located in the subdomain $D_{1}$. The $N$ points $x_{i}^{(2)}$ and the point $y_{2}$ are located in the subdomain $D_{2}$. The motion is killed with probability $1-\sum_{i=1}^{N}\left(p_{i}+q_{i}\right)$ and otherwise replaced according to the $p_{i}$ and to the $q_{i}$ at one of the points $x_{i}^{(1)}$ or $x_{i}^{(2)}$.

We are not done yet with double randomization. It will be also used inside each subdomain as our main tool to solve Poisson equations is the WOS method. Indeed, for a sphere $S(x, r)$ of radius $r$ centered at a point $x$ in $D_{1}$, we have

$$
u_{1}(x)=\mathbb{E}_{x}\left[u_{1}\left(W_{\tau_{S(x, r)}}\right) e^{-\frac{\lambda_{1}}{a_{1}} \tau_{S(x, r)}}+\int_{0}^{\tau_{S(x, r)}} \frac{1}{a_{1}} f\left(W_{t}\right) e^{-\frac{\lambda_{1}}{a_{1}} t} d t\right]
$$

where $u_{1}$ is also in the right handside of the representation. Using the simulation tools described in the next section, we will have

$$
u_{1}(x)=p u_{1}(z)+c f(y)
$$

where $0<p \leqslant 1, z$ is on the sphere $S(x, r)$ and $y$ is in its interior. The quantity $c f(y)$ is added to the score, the motion is killed with probability $1-p$ and otherwise replaced at point $z$.

In the case of the Poisson-Boltzmann equation, a Feynman-Kac formula for the solution as well as a proof of convergence of the relative algorithm have been obtained in [2]. The impact of the discretization parameters on the global bias of the solution has been studied heuristically in [16] for transmission conditions at the interface between two subdomains. The conclusion was that the global bias is the product of the local bias at the interface by the mean number of hits of the interface. This global bias will be studied in detail in section 5.1 for Robin boundary conditions. 


\section{Walk on spheres simulation and scoring}

In this section, we describe the WOS method and its associated score in dimension two and three for the Poisson equation with or without killing. We especially focus on the case with both a source term and a damping term in the equation. We also describe two versions of the Euler scheme to deal with this last situation in order to make numerical comparisons.

\subsection{Standard walk on spheres}

\subsubsection{Description of the motion}

The WOS introduced by E. M. Muller in 1956 [23] gives a very efficient way to obtain the solution at a point $z$ of the Laplace equation in a domain $D$ with Dirichlet boundary conditions. The walk starts at $z_{0}=z$ and thanks to the isotropy of the Brownian motion, the new position $z_{1}$ is drawn uniformly at random on the sphere $S_{1}$ tangent to the domain $D$. This means for example in dimension two that the angle in radial coordinate is picked uniformly at random in $[0,2 \pi]$. The algorithm stops when the $n$th position $z_{n}$ defined as previously is at a distance to the boundary of the domain smaller than a fixed parameter $\varepsilon$. The value of the boundary term at the normal projection of $z_{n}$ on the boundary gives the score of the walk. The approximate solution is the mean value of the scores of the different walks. This method is very efficient to deal with elliptic equations of Laplace type. Indeed, the average number of steps until absorption is proportional to $|\ln (\varepsilon)|$ while the error due to the projection is a $O(\varepsilon)[24]$.

\subsubsection{Conditioned Green function scoring}

When we solve the Poisson equation, we also have to compute the contribution of the source term along the walk. To compute this contribution at the $j$ th step of the walk, we use the Green function $K(z, u)$ conditioned by the exit point $z_{j+1}$ writing

$$
\mathbf{E}_{z_{j}}\left[\int_{0}^{\tau_{S_{j+1}}} f\left(W_{s}\right) d s \mid W_{\tau_{S_{j+1}}}=z_{j+1}\right]=\int_{S_{j+1}} K\left(z_{j+1}, u\right) f(u) d u .
$$

For the unit ball in dimension 2 [11], the cumulative radial distribution of this conditioned Green function is given by

$$
f_{R}(r)=r^{2}(1-2 \log (r)) 1_{0 \leqslant r \leqslant 1}
$$

while its cumulative conditional angular distribution is

$$
f_{\theta \mid R=r}(\theta)=\frac{1}{2}+\frac{1}{\pi} \arctan \left(\frac{1+r}{1-r} \frac{\tan \left(\theta-\theta_{0}\right)}{2}\right) 1_{-\pi \leqslant \theta \leqslant \pi}
$$


where $\exp \left(i \theta_{0}\right)=z$. For the ball $B_{j+1}$ of radius $r_{j+1}$ centered at $\left(x_{j}, y_{j}\right)$, we write

$$
\int_{B_{j+1}} K\left(z_{j+1}, u\right) f(u) d u=\frac{r_{j+1}^{2}}{2} \mathbf{E}\left(f\left(P_{j}\right)\right)
$$

with $P_{j}=\left(r_{j+1} R \cos (\theta)+x_{j}, r_{j+1} R \sin (\theta)+y_{j}\right)$. In dimension 3 , the conditioned Green function can also be obtained [28]. However, its cumulative distribution seems very hard to compute. Maybe some techniques based on the rejection method can be used. We prefer to use another method based on precomputed simulations that we describe in the next subsection.

\subsubsection{One random point scoring}

In dimension 3, we have just seen that it is not easy to obtain the conditional Green function. Furthermore, even when this conditional density is known analytically, one has to sample from it. In dimension two this involves for instance some iterations of the Newton method to simulate the radial distribution. We can replace this exact computation by an approximate one based on the one random point method [18]. We note first that for any starting point $x \in D$

$$
E_{x}\left(\int_{0}^{\tau_{D}} f\left(W_{s}\right) d s\right)=E_{x}\left(\tau_{D} f\left(W_{U \tau_{D}}\right)\right)
$$

where $U$ is a uniform random variable in $[0,1]$ independent of $W_{s}$. Consequently, the weak approximation of path integrals can be reduced to evaluations of the source term at one random point of the path times the lenght $\tau_{D}$ of the path. The idea is now to precompute samples of the couple $\left(\tau_{S^{(1)}}, W_{U \tau_{S^{(1)}}}\right)$ of paths starting at the center of the unit circle or the unit sphere assuming furthemore that the exit point $M$ is $(1,0)$ or $(1,0,0)$ respectively (this avoids to also store the exit point of the path). In practice, we simulate this path using the Euler scheme corrected by the half-space approximation with a very fine time step $\triangle t$. We keep in memory the first $n$ points $W_{j \triangle t}($ with $0 \leqslant j \leqslant n-1)$ of the path before absorption, the exit point $\widehat{W_{n \triangle t}}$ which is the orthogonal projection on the boundary of $W_{n \triangle t}$ and the exit time $\tau_{B}=n \triangle t$. Then, we pick uniformly at random one point $W_{i \triangle t}$ among the first $n$ points of the path and make a rotation of this point such that the exit point $\widehat{W_{n \triangle t}}$ is now $M$. In dimension two, if $\widehat{W_{n \triangle t}}=(\cos \theta, \sin \theta)$ then it is transformed into point $M(1,0)$ thanks to a rotation which matrix is

$$
\left(\begin{array}{cc}
\cos \theta & \sin \theta \\
-\sin \theta & \cos \theta
\end{array}\right)
$$

In dimension three, if $\widehat{W_{n \triangle t}}=(\cos \theta, \sin \theta \cos \varphi, \sin \theta \sin \varphi)$, then it is transformed into point $M(1,0,0)$ thanks to a rotation which matrix is

$$
\left(\begin{array}{ccc}
\cos \theta & \sin \theta \cos \varphi & \sin \theta \sin \varphi \\
-\cos \varphi \sin \theta & \sin ^{2} \varphi+\cos ^{2} \varphi \cos \theta & -\sin \varphi \cos \varphi(1-\cos \theta) \\
-\sin \varphi \sin \theta & -\sin \varphi \cos \varphi(1-\cos \theta) & \cos ^{2} \varphi+\sin ^{2} \varphi \cos \theta
\end{array}\right) .
$$


We store in a large file $N$ realizations of the couple $\left(\tau_{S^{(1)}}, W_{U \tau_{S^{(1)}}}\right)$ using this method. Thanks to scaling arguments, we have the formula

$E_{x}\left(\int_{0}^{\tau_{S(x, r)}} f\left(\sqrt{a} W_{s}\right) d s / x+r W_{\tau_{S^{(1)}}}=z\right)=E_{x}\left(\frac{r^{2}}{a} \tau_{S^{(1)}} f\left(x+r W_{U \tau_{S^{(1)}}}\right) / x+W_{\tau_{S^{(1)}}}=z\right)$

and the approximation

$$
E_{x}\left(\int_{0}^{\tau_{S(x, r)}} f\left(\sqrt{a} W_{s}\right) d s / x+r W_{\tau_{S^{(1)}}}=z\right) \simeq \frac{r^{2}}{a} \tau_{S^{(1)}} f\left(R_{z}\left(x+r W_{U \tau_{S^{(1)}}}\right)\right)
$$

where $R_{z}$ is the rotation that transforms $M$ in $\frac{z}{\|z\|}$ which is obtained by changing $\theta$ in $-\theta$ in the above formulae.

\subsection{Spherical subdomains}

We now discuss of the special situation when an inclusion in the domain is circular and there are neither a source term or a damping term in the equation. In this case, it is possible to replace directly the particle on the boundary whatever its position inside the circle is. When lying in a circular inclusion, this unbiased technique enables to reduce to one the number of steps before hitting the boundary. This uncentered walk on spheres (UWOS) has been used successfully in recent works $[2,20]$ for the three dimensional PoissonBoltzmann equation.

\subsubsection{Uncentered walk on circles}

In dimension two, thanks to the Poisson integral formula the density

$$
f_{r, \alpha}(\theta)=\frac{1}{2 \pi} \frac{R^{2}-r^{2}}{R^{2}+r^{2}-2 r R \cos (\theta-\alpha)}=\frac{r^{2}-R^{2}}{4 \pi r R} \frac{1}{\cos (\theta-\alpha)-\frac{R^{2}+r^{2}}{2 r R}}
$$

represents the law of the exit position on the circle of radius $R$ and centered at the origin of a Brownian motion starting at point $r \exp (i \alpha)$. To simulate a random variable $Y_{0}$ with density $f_{r, 0}(\theta)$, we let

$$
\beta=\frac{r^{2}-R^{2}}{4 \pi r R}, \gamma=-\frac{\left(R^{2}+r^{2}\right)}{2 r R}
$$

and first compute the cumulative distribution

$$
F_{r, 0}(t)=\int_{0}^{t} \frac{\beta}{\gamma+\cos (\theta)} d \theta=\frac{2 \beta \arctan \left(\frac{\left.(-1+\gamma) \tan \left(\frac{t}{2}\right)\right)}{\sqrt{\gamma^{2}-1}}\right)}{\sqrt{\gamma^{2}-1}} .
$$

Furthermore, as $\frac{2 \beta}{\sqrt{\gamma^{2}-1}}=-\frac{1}{\pi}$ and $\frac{-1+\gamma}{\sqrt{\gamma^{2}-1}}=-\frac{R+r}{R-r}$, we obtain that

$$
Y_{0}=F_{r, 0}^{-1}(U)=2 \arctan \left(\frac{R-r}{R+r} \tan (\pi U)\right),
$$

where $U$ is a uniform random variable on $[0,1]$. As $f_{r, \alpha}(\theta)=f_{r, 0}(\theta-\alpha)$, the random variable $Y_{\alpha}=Y_{0}+\alpha$ admits $f_{r, \alpha}(\theta)$ for density. 


\subsubsection{Uncentered walk on spheres}

In dimension three, we assume without loss of generality that the starting point is $x=(r, 0,0)$ and that the domain is the sphere of radius $R$ centered at the origin. First the coordinates of the exit point are

$$
\left\{\begin{array}{l}
x_{1}=R \cos \theta \sin \varphi \\
x_{2}=R \sin \theta \sin \varphi \\
x_{3}=R \cos \varphi
\end{array}\right.
$$

where $\theta$ has a uniform law in $[0,2 \pi]$. Then, thanks to the Poisson integral formula, the density of the angle $\varphi$ writes

$$
f_{R, r}(\varphi)=\frac{\left(R^{2}-r^{2}\right) R^{2} \sin \varphi}{4 \pi R\left(R^{2}-2 R r \cos \varphi+r^{2}\right)^{3 / 2}}
$$

It is easy to compute its cumulative distribution function by

$$
F_{R, r}(\varphi)=\frac{R^{2}-r^{2}}{2 \pi R r}\left(\frac{R}{R-r}-\frac{R}{\sqrt{R^{2}-2 R r \cos \varphi+r^{2}}}\right)
$$

Giving now a uniform random variable $U$ independent of $\theta$, the random variable

$$
Y_{\varphi}=F_{R, r}^{-1}(U)=\arccos \left(\frac{R^{2}+r^{2}}{2 \pi R r}-\frac{1}{\pi 2 R r}\left(\frac{R^{2}-r^{2}}{R+r-2 r U}\right)^{2}\right)
$$

$\operatorname{admits} f_{R, r}(\varphi)$ for density.

\subsection{Simulations involving the damping term}

In this section, we explain how to approximate the representation

$$
u(x)=\mathbb{E}_{x}\left[g\left(W_{\tau_{D}}\right) e^{-\lambda \tau_{D}}+\int_{0}^{\tau_{D}} f\left(W_{t}\right) e^{-\lambda t} d t\right]
$$

using simulation schemes. We first use a basic version based on the Euler scheme. Afterwards, we describe how to interpret this scheme and the WOS method as random walks with a killing rate.

\subsubsection{Euler scheme simulations}

The standard approximation of

$$
g\left(W_{\tau_{D}}\right) \exp \left(-\lambda \tau_{D}\right)+\int_{0}^{\tau_{D}} f\left(W_{s}\right) \exp (-\lambda s) d s
$$

using the Euler scheme coupled with the rectangle method writes

$$
S=g\left(\widetilde{W_{n \triangle t}}\right) \exp (-\lambda n \triangle t)+\triangle t \sum_{i=0}^{n-1} f\left(W_{i \triangle t}\right) \exp (-\lambda i \triangle t)
$$


with $W_{0}=x$ and $W_{(n+1) \triangle t}=W_{n \triangle t}+\sqrt{\triangle t} Z_{n+1}$ where the $Z_{n}$ are standard independent normal random variables. The absorption at point $\widetilde{W_{n \triangle t}}$ of the boundary occurs between step $n-1$ and $n$. This point is the orthogonal projection of $W_{n \triangle t}$ on the boundary. We also use the half space approximation which says that the Euler scheme stops between steps $j$ and $j+1$ with probability $\exp \left(-\frac{2 d_{j} d_{j+1}}{\triangle t}\right)$ where $d_{j}$ is the distance from point $W_{j \triangle t}$ to the boundary of $D$.

We can give another interpretation of the score $S$ of a given walk by computing it recursively. Letting $Y_{0}=0$, we define

$$
Y_{j+1}=\exp (-\lambda \triangle t) Y_{j}+b_{j}
$$

with $b_{j}=\Delta t f\left(W_{j \triangle t}\right)$ for $0 \leqslant j \leqslant n-1$ and with $b_{n}=g\left(\widetilde{\left.W_{n \triangle t}\right)}\right.$. Consequently $Y_{j+1}=E\left(X_{j} Y_{j}\right)+b_{j}$ where the independent Bernoulli random variables $X_{j}$ are such that $P\left(X_{j}=1\right)=\exp (-\lambda \triangle t)$. We obviously have $E\left(Y_{n+1}\right)=S$.

From the simulation point of view, at each step $j$ we stop the walk with probability $1-\exp (-\lambda \triangle t)$ and add $b_{j}$ to its score. We can sample from $Y_{n+1}$ only once because the sum $S$ itself is also a random variable. This corresponds to a Monte Carlo approximation on a product space.

\subsubsection{WOS with a killing rate (KWOS)}

The WOS method is based on the same idea than the last algorithm. At a given step $j$ of the walk, we write

$$
u\left(x_{j}\right)=E_{x_{j}}\left(u\left(W_{\tau_{S_{j}}}\right) \exp \left(-\lambda \tau_{S_{j}}\right)+\int_{0}^{\tau_{S_{j}}} f\left(W_{s}\right) \exp (-\lambda s) d s\right)
$$

where $S_{j}$ is the sphere centered at $x_{j}$ with radius $r_{j}=d\left(x_{j}, \partial D\right)$. We have

$$
E_{x_{j}}\left(u\left(W_{\tau_{S_{j}}}\right) \exp \left(-\lambda \tau_{S_{j}}\right)\right)=E_{x_{j}}\left(u\left(W_{\tau_{S_{j}}}\right) E_{x_{j}}\left(\exp \left(-\lambda \tau_{S_{j}}\right)\right)\right.
$$

thanks to the independence between the exit position and the exit time of the Brownian motion from a sphere. The Laplace transform $E_{x_{j}}\left(\exp \left(-\lambda \tau_{S_{j}}\right)\right)$ is the survival probability which plays the same role than the parameter $\exp (-\lambda \triangle t)$ for the Euler scheme. It depends in addition on the radius $r_{j}$ and is equal to $\frac{\sqrt{2 \lambda} r_{j}}{\sinh \left(\sqrt{2 \lambda} r_{j}\right)}$ in dimension three and to $\frac{1}{I_{0}\left(\sqrt{2 \lambda} r_{j}\right)}$ in dimension two where $I_{0}(x)=$ $\sum_{k=0}^{\infty} \frac{\left(\frac{x}{2}\right)^{2 k}}{(k !)^{2}}$. If the walk reaches the aborption boundary layer, then the WOS approximation of $g\left(W_{\tau_{D}}\right)$ is added to the score of the walk.

It remains to explain how we can compute the contribution of the source term

$$
\left.\int_{0}^{\tau_{S_{j}}} f\left(W_{s}\right) \exp (-\lambda s) d s\right)
$$

in the sphere $S_{j}$. For the unit sphere $S^{(1)}$, its approximation for a given path by the rectangle method writes

$$
\triangle t \sum_{i=0}^{n-1} f\left(W_{i \triangle t}\right) \exp (-\lambda i \triangle t)=\triangle t \frac{1-\exp (-n \lambda \triangle t)}{1-\exp (-\lambda \triangle t)} E\left(f\left(W_{J \triangle t}\right)\right)
$$


where $J \in[0, n-1]$ is a discrete random variable such that

$$
P(J=i)=\exp (-\lambda i \triangle t) \frac{1-\exp (-\lambda \triangle t)}{1-\exp (-n \lambda \triangle t)} .
$$

Hence, we can approximate the source term by

$$
\triangle t \frac{1-\exp (-n \lambda \triangle t)}{1-\exp (-\lambda \triangle t)} f\left(W_{J \triangle t}\right)
$$

using only one point of the trajectory picked according to the law of $J$. Thanks to scaling arguments, for a sphere of radius $r$ centered at $x$ the approximation of the source term simply writes

$$
\triangle t \frac{1-\exp \left(-n \lambda r^{2} \triangle t\right)}{1-\exp \left(-\lambda r^{2} \triangle t\right)} r^{2} f\left(x+r W_{J_{r} \triangle t}\right)
$$

where $J_{r} \in[0, n-1]$ is a discrete random variable such that

$$
P\left(J_{r}=i\right)=\exp \left(-\lambda r^{2} i \triangle t\right) \frac{1-\exp \left(-\lambda r^{2} \triangle t\right)}{1-\exp \left(-n \lambda r^{2} \triangle t\right)} .
$$

The law of $J_{r}$ is not uniform and more importantly depends on the radius of the sphere. We can simulate $J_{r}$ by inverting its discrete distribution function which lead to

$$
J_{r}=1+\left\lfloor\frac{-1}{\lambda r^{2} \Delta t} \ln \left(1-U+U e^{-n \lambda r^{2} \Delta t}\right)\right\rfloor
$$

where $U$ is a uniform random variable on $[0,1]$. Hence if we want to use this approximation, we need to precompute and store not only one random point of the discretized Brownian motion killed at the boundary of the unit sphere like in section but all the points of the path. For the numerical results of section 5 , we will store $10^{4}$ discretized trajectories with a step size $\triangle t=10^{-3}$.

\section{Stochastic finite differences}

The aim of this section is to provide order three approximations of Robin and transmissions boundary conditions based on stochastic finite differences techniques. These techniques are the extensions to more complex situations and to the three-dimensional case of the recent works [16, 17]. For the transmission conditions, the computations are done on a domain $D$ divided in two subdomains $D_{1}$ and $D_{2}$ with a common boundary and for the Robin boundary conditions on a part of the boundary. The normal vector to this boundary is colinear to the first coordinate. More precisely, we assume that these boundaries are hitten at point $(0,0)$ in dimension two and point $(0,0,0)$ in dimension three. In the case of Robin conditions, the interior of the domain corresponds to the points $x$ such that $x_{1}>0$. In the case of transmission conditions, the subdomain $D_{1}$ corresponds to the points $x$ such that $x_{1}>0$. 


\subsection{Robin boundary conditions without damping}

We first consider the Poisson equation with Robin boundary conditions in a domain $\mathrm{D}$ with boundary $\partial D$

$$
\left\{\begin{array}{c}
-\frac{1}{2} \Delta u(x)=f(x) \quad x \in D \\
\alpha(x) u(x)+\beta(x) \frac{\partial u(x)}{\partial n}=g(x) \quad x \in \partial D
\end{array}\right.
$$

where $\alpha$ and $\beta$ are positive parameters that may depend on $x$. In fact, as we only look at what happens at one given point of the boundary, we just denote by $\alpha$ and $\beta$ their value at this particular point.

\subsubsection{Dimension two approximation}

We define

$$
\Delta^{h} u(0,0)=\frac{u(h, 0)+u(-h, 0)+u(0, h)+u(0,-h)-4 u(0,0)}{h^{2}}
$$

which is a discrete approximation of the Laplace operator $\Delta u$ and

$$
\bar{\nabla}_{x_{1}}^{h} u(0,0)=\frac{4 u(h, 0)-3 u(0,0)-u(2 h, 0)}{2 h}
$$

which is an approximation of its normal derivative with respect to $x_{1}$.

Given a discretization step $h>0$, the finite differences approximation of the Laplace operator of a $\mathcal{C}^{3}$ function at point $(h, 0)$ leads to

$$
-\frac{1}{2} \Delta^{h} u(h, 0) \simeq f(h, 0)
$$

while the approximation of its normal derivative at point $(h, 0)$ to

$$
\alpha u(0,0)+\beta\left(-\bar{\nabla}_{x_{1}}^{h} u(0,0)\right) \simeq g(0,0) .
$$

If we now multiply equation (4.2) by $2 \beta h^{2}$, equation (4.3) by $2 h$ and that we sum the resulting equations, the approximation of the solution at point $(0,0)$ writes

$$
u(0,0) \simeq \frac{\beta(u(h,-h)+u(h, h))}{2 \alpha h+2 \beta}+\frac{h}{\alpha h+\beta} g(0,0)+\frac{\beta h^{2}}{\alpha h+\beta} f(h, 0) .
$$

The approximation error of this formula is a $O\left(h^{3}\right)$. All the approximations developed similarly in the following will have the same approximation order. Formula (4.4) is usefull for Monte Carlo simulations. The quantity

$$
\frac{\beta}{2 \alpha h+2 \beta}(u(h,-h)+u(h, h))
$$


is the mean value of a random variable that takes the values $u(h,-h)$ and $u(h, h)$ with probability $\frac{\beta}{2 \alpha h+2 \beta}$ and 0 with probability $\frac{\alpha h}{\alpha h+\beta}$. More precisely, when the Brownian motion hits the boundary, the quantity

$$
\frac{h}{\alpha h+\beta} g(0,0)+\frac{\beta h^{2}}{\alpha h+\beta} f(h, 0)
$$

is added to the score. It is killed with probability $\frac{\alpha h}{\alpha h+\beta}$, otherwise the walk restarts equiprobably at one of the two points

$$
(h,-h),(h, h) \text {. }
$$

These Robin conditions are hence a mix between the Dirichlet conditions where the particle is killed when hitting the boundary and Neumann conditions where it is purely reflected. When close to the other boundaries the replacement point may lie outside the domain. In this case, we reduce iteratively $h$ by a factor two until it lies into it.

\subsubsection{Dimension three approximation}

We use the same kind of approximations than in dimension two. The only difference is that we need two different steps $h$ et $\gamma h$ to build our finite differences schemes. We now let

$$
\begin{aligned}
& A_{x}=\frac{-u(2 h, 0,0)-u(0,0,0)+2 u(h, 0,0)}{2 h^{2}}, \\
& A_{y}=\frac{-u(h, \gamma h, z)-u(h,-\gamma h, 0)+2 u(h, 0,0)}{2 \gamma^{2} h^{2}}, \\
& A_{z}=\frac{-u(h, 0, \gamma h)-u(h, 0,-\gamma h)+2 u(h, 0,0)}{2 \gamma^{2} h^{2}} .
\end{aligned}
$$

The approximations of the Laplace operator at point $(h, 0,0)$ and of its normal derivative at point $(0,0,0)$ lead to the equations

$$
A_{x}+A_{y}+A_{z} \simeq f(h, 0,0)
$$

and

$$
\alpha u+\beta \frac{\partial u}{\partial n} \simeq \alpha u+\beta \frac{3 u(0,0,0)+u(2 h, 0,0)-4 u(h, 0,0)}{2 h}=g(0,0,0) .
$$

We now multiply equation (4.5) by $2 \beta h^{2}$, equation (4.6) by $2 h$ and sum the resulting equations. To get rid of the terms $u(2 h, 0,0)$ and $u(h, 0,0)$ simultaneously, we need to choose $\gamma=\sqrt{2}$. We finally obtain

$$
u(0,0,0) \simeq \frac{\beta P^{h, \sqrt{2}} u}{\alpha h+\beta}+\frac{h}{\alpha h+\beta} g(0,0,0)+\frac{\beta h^{2}}{\alpha h+\beta} f(h, 0,0)
$$


where

$$
P^{h, \gamma} u=\frac{u(h, 0,-\gamma h)+u(h, 0, \gamma h)+u(h, \gamma h, 0)+u(h,-\gamma h, 0)}{2 \gamma^{2}} .
$$

The motion is killed with probability $\frac{h}{\alpha h+\beta}$ and is replaced equiprobably at one of the four points

$$
\{(h, 0, \sqrt{2} h),(h, 0,-\sqrt{2} h),(h, \sqrt{2} h, 0),(h,-\sqrt{2} h, 0)\}
$$

with the complementary probability.

\subsection{Robin boundary conditions with damping}

We now consider the equation with a damping term

$$
\left\{\begin{array}{c}
-\frac{1}{2} \Delta u(x)+\lambda u(x)=f(x), \quad x \in D \\
\alpha u(x)+\beta \frac{\partial u(x)}{\partial n}=g(x) \quad x \in \partial D
\end{array}\right.
$$

where $\lambda$ is a positive constant.

\subsubsection{Dimension two approximation}

Even in dimension two, we now need two different steps $h$ et $\gamma h$ to take into account the damping term. The approximation of the source term leads to

$$
\begin{array}{r}
\frac{-u(2 h, 0)-u(0,0)+2 u(h, 0)}{2 h^{2}}+\frac{-u(h, \gamma h)-u(h,-\gamma h)+2 u(h, 0)}{2 \gamma^{2} h^{2}} \\
+\lambda u(h, 0) \simeq f(h, 0) .
\end{array}
$$

Multiplying equation (4.10) by $2 \beta \gamma^{2} h^{2}$, equation (4.3) by $2 h \gamma^{2}$ and summing the resulting equations, we obtain

$$
\begin{aligned}
& 2 \gamma^{2}(\alpha h+\beta) u(0,0)-\beta(u(h, \gamma h)+u(h,-\gamma h))+\left(2 \beta+2 \beta \lambda \gamma^{2} h^{2}-2 \gamma^{2} \beta\right) u(h, 0) \\
& =2 \gamma^{2} \beta h^{2} f(h, 0)+2 h \gamma^{2} g(0,0) .
\end{aligned}
$$

To get rid of the term $u(h, 0)$, we choose $\gamma=\sqrt{\frac{1}{1-\lambda h^{2}}}$ for $h$ small enough such that $\frac{1}{1-\lambda h^{2}}>0$. This leads finally to the approximation

$$
u(0,0) \simeq \frac{\beta(u(h, \gamma h)+u(h,-\gamma h))}{2 \gamma^{2}(\alpha h+\beta)}+\frac{h}{\alpha h+\beta} g(0,0)+\frac{\beta h^{2}}{\alpha h+\beta} f(h, 0) .
$$

The quantity

$$
\frac{h}{\alpha h+\beta} g(0,0)+\frac{\beta h^{2}}{\alpha h+\beta} f(h, 0)
$$

is added to the score. The motion is killed with probability $1-\frac{\beta}{\gamma^{2}(\alpha h+\beta)}$, otherwise it is replaced equiprobably at one of the two points

$$
(h, \gamma h),(h,-\gamma h) .
$$




\subsubsection{Dimension three approximation}

Similarly as for the two-dimensional case, we use the step $h$ for the first coordinate and the step $\gamma h$ for the two others. Thanks to analogous computations, we obtain the approximation

$$
u(0,0,0) \simeq \frac{\beta}{\alpha h+\beta} P^{h, \gamma} u \frac{h}{\alpha h+\beta} g(0,0,0)+\frac{\beta h^{2}}{\alpha h+\beta} f(h, 0,0)
$$

where $\gamma=\sqrt{\frac{2}{1-\lambda h^{2}}}$ with some obvious constraints on $h$. The quantity

$$
\frac{h}{\alpha h+\beta} g(0,0,0)+\frac{\beta h^{2}}{\alpha h+\beta} f(h, 0,0)
$$

is added to the score. The motion is killed with probability $1-\frac{\beta}{\gamma^{2}(\alpha h+\beta)}$ otherwise it continues equiprobably at one of the four points

$$
(h, \gamma h, 0),(h,-\gamma h, 0),(h, 0, \gamma h),(h, 0,-\gamma h) .
$$

\subsection{Transmission conditions without damping}

We first consider the divergence form equation with Dirichlet boundary conditions

$$
\left\{\begin{array}{c}
\frac{1}{2} \nabla(a(x) \nabla u(x))=-f(x), \quad x \in D \\
u(x)=g(x), \quad x \in \partial D
\end{array}\right.
$$

in a domain $D=D_{1} \cup D_{2}$ such that the scalar coefficient $a(x)$ verifies

$$
\begin{cases}a(x)=a_{1} & x \in D_{1} \\ a(x)=a_{2} & x \in D_{2}\end{cases}
$$

\subsubsection{Dimension two approximation}

We recall the method developed in [16]. The crucial point is that

$$
\frac{h^{2}}{2} \triangle^{h} u(h, 0)=-h \bar{\nabla}_{x_{1}}^{h} u(0,0)-u(0,0)+P^{h} u(0,0)
$$

where

$$
P^{h} u=\frac{u(h, h)+u(h,-h)}{2} .
$$

If $u$ solves (4.12), then $u$ satisfies the transmission condition

$$
a_{1} \bar{\nabla}_{x_{1}}^{h_{1}} u(0,0)=a_{2} \bar{\nabla}_{x_{1}}^{-h_{2}} u(0,0)+O\left(a_{2} h_{2}^{2}+a_{1} h_{1}^{2}\right)
$$

where $h_{1}$ and $h_{2}$ are the finite differences steps for one side or the other of the interface. These two steps can be different but we assume that they are always 
proportional so that all the following approximations are still exact up to a $O\left(h_{1}^{3}\right)$. Thanks to equations (4.14) and (4.15), we obtain the approximation

$$
u(0,0) \simeq p_{1} P^{h_{1}} u+p_{2} P^{-h_{2}} u+C_{1} f\left(h_{1}, 0\right)+C_{2} f\left(-h_{2}, 0\right)
$$

where

and

$$
p_{1}=\frac{a_{1} h_{2}}{a_{1} h_{2}+a_{2} h_{1}}, p_{2}=\frac{a_{2} h_{1}}{a_{1} h_{2}+a_{2} h_{1}}
$$

$$
C_{1}=\frac{h_{1}^{2} h_{2}}{a_{1} h_{2}+a_{2} h_{1}}, C_{2}=\frac{h_{1} h_{2}^{2}}{a_{1} h_{2}+a_{2} h_{1}} .
$$

In terms of simulation, this means that with probability $p_{1}$ the motion is replaced in $D_{1}$ equibrobably at point $\left(h_{1},-h_{1}\right)$ or at point $\left(h_{1}, h_{1}\right)$ and with probability $p_{2}$ in $D_{2}$ equiprobably at point $\left(-h_{2},-h_{2}\right)$ or at point $\left(-h_{2}, h_{2}\right)$. The quantity

$$
C_{1} f\left(h_{1}, 0\right)+C_{2} f\left(-h_{2}, 0\right)
$$

is added to the score.

\subsubsection{Dimension three approximation}

In dimension three, we use the same tools than in dimension two replacing just $P^{h} u$ by $P^{h, \sqrt{2}} u$ in relation (4.16). We obtain

$$
u(0,0,0) \simeq p_{1} P^{h_{1}, \sqrt{2}} u+p_{2} P^{-h_{2}, \sqrt{2}}+C_{1} f\left(h_{1}, 0,0\right)+C_{2} f\left(-h_{2}, 0,0\right)
$$

where $P^{h, \sqrt{2}}$ is defined in (4.8). The probabilities to go into each subdomain are the same than in dimension two and the four replacement points in each subdomains are respectively

$$
\left\{\left(h_{1}, 0, \sqrt{2} h_{1}\right),\left(h_{1}, 0,-\sqrt{2} h_{1}\right),\left(h_{1}, \sqrt{2} h_{1}, 0\right),\left(h_{1},-\sqrt{2} h_{1}, 0\right)\right\}
$$

in $D_{1}$ and

$$
\left\{\left(-h_{2}, 0, \sqrt{2} h_{2}\right),\left(-h_{2}, 0,-\sqrt{2} h_{2}\right),\left(-h_{2}, \sqrt{2} h_{2}, 0\right),\left(-h_{2},-\sqrt{2} h_{2}, 0\right)\right\}
$$

in $D_{2}$. The quantity

$$
C_{1} f\left(h_{1}, 0,0\right)+C_{2} f\left(-h_{2}, 0,0\right)
$$

is added to the score.

\subsection{Transmission conditions with damping}

We now consider the equation

$$
\left\{\begin{array}{c}
-\frac{1}{2} \nabla(a(x) \nabla u(x))+\lambda(x) u(x)=f(x), x \in D \\
u(x)=g(x), x \in \partial D
\end{array}\right.
$$

where

$$
\begin{cases}a(x)=a_{1}, \lambda(x)=\lambda_{1} & x \in D_{1} \\ a(x)=a_{2}, \lambda(x)=\lambda_{2} & x \in D_{2}\end{cases}
$$




\subsubsection{Dimension two approximation}

To obtain our replacement formula, we write the approximations

$$
\begin{aligned}
& a_{1}\left(\frac{-u\left(2 h_{1}, 0\right)-u(0,0)+2 u\left(h_{1}, 0\right)}{2 h_{1}^{2}}+\frac{-u\left(h_{1}, \gamma_{1} h_{1}\right)-u\left(h_{1},-\gamma_{1} h_{1}\right)+2 u\left(h_{1}, 0\right)}{2 \gamma_{1}^{2} h_{1}^{2}}\right) \\
& +\lambda_{1} u\left(h_{1}, 0\right) \simeq f\left(h_{1}, 0\right)
\end{aligned}
$$

in domain $D_{1}$ and

$$
\begin{aligned}
& a_{2}\left(\frac{-u\left(-2 h_{2}, 0\right)-u(0,0)+2 u\left(-h_{2}, 0\right)}{2 h_{2}^{2}}+\frac{-u\left(h_{2}, \gamma_{1} h_{2}\right)-u\left(-h_{2},-\gamma_{2} h_{2}\right)+2 u\left(-h_{2}, 0\right)}{2 \gamma_{2} h_{2}}\right) \\
& +\lambda_{2} u\left(-h_{2}, 0\right) \simeq f\left(-h_{2}, 0\right)
\end{aligned}
$$

in domain $D_{2}$ with different finite differences parameters $h_{1}$ and $h_{2}$ and two constants $\gamma_{1}$ and $\gamma_{2}$. The approximation of the flux conditions leads to

$a_{1} \frac{\left(4 u\left(h_{1}, 0\right)-3 u(0,0)-u\left(2 h_{1}, 0\right)\right)}{2 h_{1}} \simeq-a_{2} \frac{\left(4 u\left(-h_{2}, 0\right)-3 u(0,0)-u\left(-2 h_{2}, 0\right)\right)}{2 h_{2}}$.

We now plug in the above equation the approximations of

$$
-u\left(2 h_{1}, 0\right)-u(0,0),-u\left(-2 h_{2}, 0\right)-u(0,0)
$$

obtained by two previous formulae and choose

$$
\gamma_{1}=\sqrt{\frac{1}{1-\frac{\lambda_{1} h_{1}^{2}}{a_{1}}}}, \gamma_{2}=\sqrt{\frac{1}{1-\frac{\lambda_{2} h_{2}^{2}}{a_{2}}}}
$$

such that the relative coefficients of the terms $u\left(h_{1}, 0\right)$ and $u\left(-h_{2}, 0\right)$ vanish. This leads to the approximation

$u(0,0) \simeq p_{1}\left(1-\frac{\lambda_{1} h_{1}^{2}}{a_{1}}\right) Q^{h_{1}, \gamma_{1}} u+p_{2}\left(1-\frac{\lambda_{2} h_{2}^{2}}{a_{2}}\right) Q^{-h_{2}, \gamma_{2}} u+C_{1} f\left(h_{1}, 0\right)+C_{2} f\left(-h_{2}, 0\right)$

where

$$
Q^{h, \gamma} u=\frac{u(h, \gamma h)+u(h,-\gamma h)}{2}
$$

and $p_{1}, p_{2}, C_{1}, C_{2}$ are defined as in 4.3.1. As in the case of transmission conditions without damping, the motion goes to $D_{1}$ with probability $p_{1}$ and it is replaced equibrobably at point $\left(h_{1},-\gamma_{1} h_{1}\right)$ or at point $\left(h_{1}, \gamma_{1} h_{1}\right)$ but now with probability $1-\frac{\lambda_{1} h_{1}^{2}}{a_{1}}$. It has a probability $\frac{\lambda_{1} h_{1}^{2}}{a_{1}}$ to stop conditionaly to going in $D_{1}$. Similarly, the motion goes to $D_{2}$ with probability $p_{2}$ and it is replaced equibrobably at point $\left(h_{2},-\gamma_{2} h_{2}\right)$ or at point $\left(h_{2}, \gamma_{2} h_{2}\right)$ but now with probability $1-\frac{\lambda_{2} h_{2}^{2}}{a_{2}}$. It has a probability $\frac{\lambda_{2} h_{2}^{2}}{a_{2}}$ to stop conditionaly to going in $D_{2}$. 


\subsubsection{Dimension three approximation}

Thanks to similar computations, we obtain the approximation

$u(0,0,0) \simeq p_{1}\left(1-\frac{\lambda_{1} h_{1}^{2}}{a_{1}}\right) E^{h_{1}, \gamma_{1}} u+p_{2}\left(1-\frac{\lambda_{2} h_{2}^{2}}{a_{2}}\right) E^{-h_{2}, \gamma_{2}} u+C_{1} f\left(h_{1}, 0,0\right)+C_{2} f\left(-h_{2}, 0,0\right)$,

where

$$
\gamma_{1}=\sqrt{\frac{2}{1-\frac{\lambda_{1} h_{1}^{2}}{a_{1}}}}, \gamma_{2}=\sqrt{\frac{2}{1-\frac{\lambda_{2} h_{2}^{2}}{a_{2}}}}
$$

and

$$
E^{h, \gamma} u=\frac{u(h, \gamma h, 0)+u(h,-\gamma h, 0)+u(h, 0, \gamma h)+u(h, 0,-\gamma h)}{4} .
$$

\section{Numerical results}

\subsection{Robin problem without source term}

Our first example is the equation

$$
\left\{\begin{array}{c}
-\frac{1}{2} \Delta u(x)=0, x \in B_{1} \\
u(x)+\beta \frac{\partial u(x)}{\partial n}=g(x), x \in \partial B_{1}
\end{array}\right.
$$

where $B_{1}$ is the unit ball and

$$
g\left(x_{1}, x_{2}\right)=-\beta x_{1} e^{x_{2}}\left(\sin x_{1}+\cos x_{1}\right)+e^{x_{2}}\left(\beta x_{2}+1\right)\left(\cos x_{1}-\sin x_{1}\right) .
$$

The exact solution of this equation is $u\left(x_{1}, x_{2}\right)=\left(\cos x_{1}-\sin x_{1}\right) e^{x_{2}}$. We make a detailed description of the algorithm based on the UWOS method of section 3.2 used to solve this equation and we analyse its bias. We also make a short comparison with the version based on the WOS method.

\subsubsection{Description of the algorithm}

We describe how to compute the solution $u\left(x_{1}, x_{2}\right)$ using the UWOS method coupled with the stochastic finite differences techniques. The algorithm 1 gives the evolution of the score of a single walk using a step $h$ for the finite differences. One needs obviously to make the average of such scores to obtain the approximation.

\subsubsection{Bias analysis}

The UWOS is an exact method to simulate the exit position of a Brownian motion from a ball. Hence the bias of the method comes only from the stochastic finite differences when hitting the boundary. Each time the Brownian motion hits the boundary, it is killed with probability $\frac{h}{h+\beta}$ and the finite differences 
Data: A point $x=\left(x_{1}, x_{2}\right) \in B_{1}$, Parameters: $h, \beta$

Set Test $\leftarrow 0$ and Score $\leftarrow 0$;

while Test $\neq 1$ do

$r=\sqrt{x_{1}^{2}+x_{2}^{2}}, \alpha=\arccos \left(\frac{x_{1}}{r}\right)$

Generate a uniform random variate $U$ on $[0,1]$

$\theta \leftarrow \alpha+2 \arctan \left(\frac{1-r}{1+r} \tan (\pi U)\right)$

$\left(x_{1}, x_{2}\right) \leftarrow(\cos \theta, \sin \theta)$

Score $\leftarrow$ Score $+\frac{h}{h+\beta} g\left(x_{1}, x_{2}\right)$

Generate a uniform random variate $U_{1}$ on $[0,1]$

if $U_{1} \leqslant \frac{h}{h+\beta}$ then

I Test $\leftarrow 1$

else

Generate a uniform random variate $U_{2}$ on $[0,1]$

$\xi_{1} \leftarrow 1-h$

if $U_{2}<0.5$ then

$\xi_{2} \leftarrow h$

else

| $\xi_{2} \leftarrow-h$

end

$x_{1} \leftarrow \xi_{1} \cos \theta-\xi_{2} \sin \theta$

$x_{2} \leftarrow \xi_{1} \sin \theta+\xi_{2} \cos \theta$

end

end

Algorithm 1: UWOS algorithm for the unit ball.

approximation adds a bias which is a $O\left(h^{3}\right)$. To compute the global bias, we need to know the mean number of hits $H$ of the boundary. As $H$ follows a geometric distribution with parameter $\frac{h}{h+\beta}$, we have

$$
\mathbf{E}(H)=\sum_{k=1}^{\infty}\left(1-\frac{h}{h+\beta}\right)^{k-1} \frac{h}{h+\beta}=1+\frac{\beta}{h} .
$$

Consequently, the mean number of hits of the boundary is a $O\left(\frac{\beta}{h}\right)$ and the global bias is a $O\left(\beta h^{2}\right)$. In figure 1, we plot in a logarithm scale the error at point $(0.5,0.5)$ as a function of the discretization step $h$ for three different values of $\beta$. The number of simulations is chosen large enough so that this error is very close to the bias of our method. The order of the method given by a linear least-square fitting of the data at four reference points is respectively $(1.9,2.01,2.08)$ for $\beta=(0.05,0.1,0.2)$. The bias also increases when $\beta$ increases. This confirms our estimation of the global bias of the method.

\subsubsection{Comparison between WOS and UWOS}

In table 1, we compare the error and the CPU times of the WOS and of the UWOS methods for the computation of the solution still at point $(0.5,0.5)$ and 


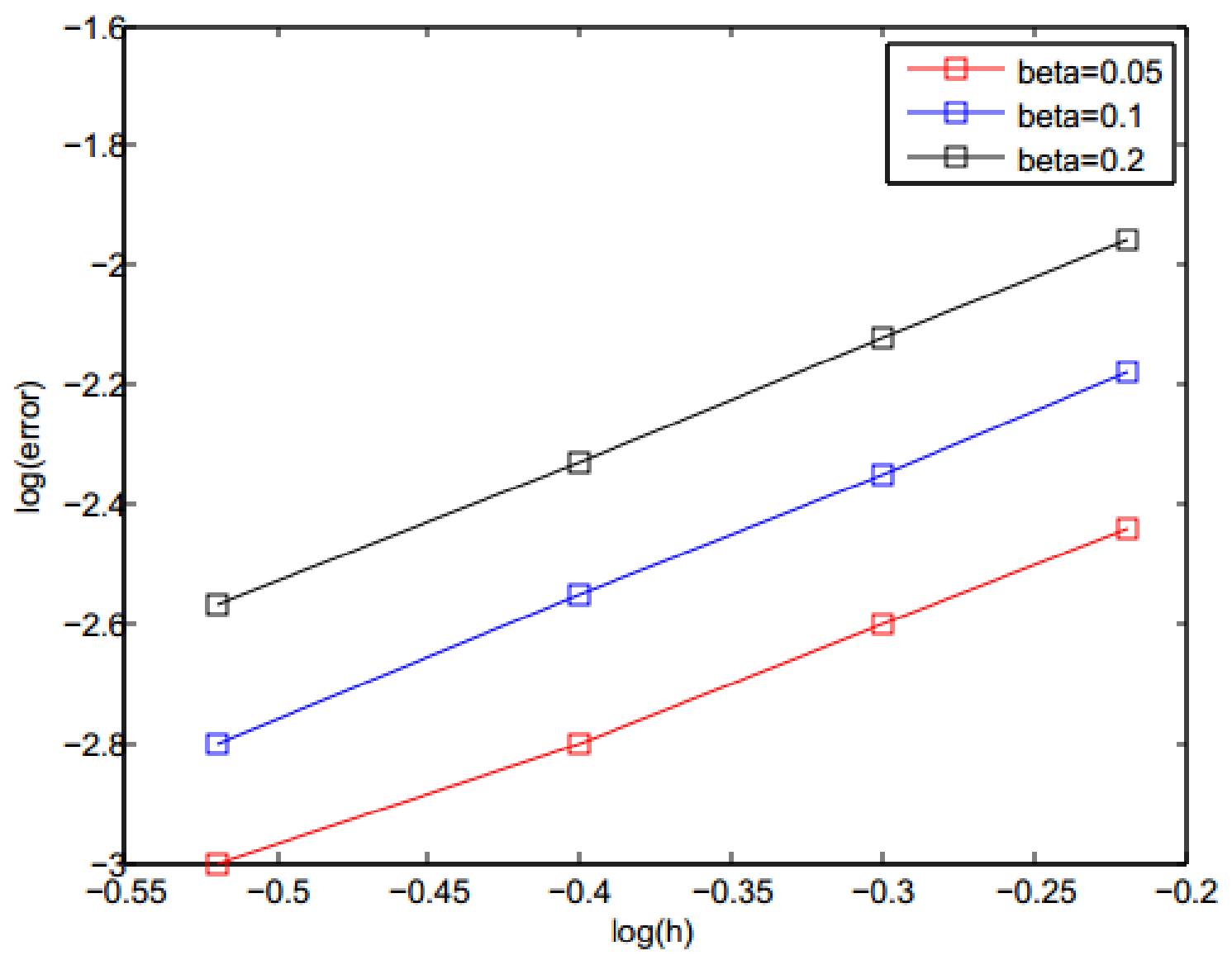

Figure 1: Bias versus step $h$ in log scale

with $\beta=0.1$. The number of simulations $N=10^{7}$ and the absorption layer $\varepsilon=10^{-6}$ are chosen so that the variance and the bias due to the layer are small. We observe that the error of the two methods are very close and that the UWOS method is three times faster. We can conclude that the method is still efficient when using WOS and that one should use the UWOS whenever it is possible.

\begin{tabular}{|c|cc|rr|}
\hline $\mathrm{h}$ & \multicolumn{2}{|c|}{ WOS } & \multicolumn{2}{c|}{ UWOS } \\
& Error & CPU & Error & CPU \\
\hline 0.3 & 0.0015 & 35 & 0.0018 & 10.3 \\
0.4 & 0.0026 & 33 & 0.0028 & 9.6 \\
0.5 & 0.0041 & 31 & 0.0045 & 9 \\
\hline
\end{tabular}

Table 1: Parameters: $\left(x_{1}, x_{2}\right)=(0.5,0.5), N=10^{7}, \beta=0.1, \varepsilon=10^{-6}$ 


\subsection{Robin problem with a source term}

\subsubsection{Robin problem without damping}

Our next equation with an additional source term is

$$
\left\{\begin{aligned}
-\frac{1}{2} \Delta u\left(x_{1}, x_{2}\right) & =-6\left(x_{1}^{2}+x_{2}^{2}\right),\left(x_{1}, x_{2}\right) \in B_{1} \\
u\left(x_{1}, x_{2}\right)+\beta \frac{\partial u\left(x_{1}, x_{2}\right)}{\partial n} & =(1+4 \beta)\left(x_{1}^{4}+x_{2}^{4}\right),\left(x_{1}, x_{2}\right) \in \partial B_{1}
\end{aligned}\right.
$$

and its exact solution is $u\left(x_{1}, x_{2}\right)=x_{1}^{4}+x_{2}^{4}$. In table 2 , we compare the error and the CPU times of the WOS using either an exact simulation of the contribution of the source term (section 3.1.2) or its approximation by the one random point method (section 3.1.3) using the same parameters than in section 5.1.3. We observe that both methods give a similar accuracy and that the one random point method gives slightly smaller CPU times. The approximate orders of two methods given by a linear least-square fitting of the data at our four reference points are respectively $(2.08,1.99)$ for the exact method and the approximate one. This confirms the efficiency of our algorithm and our bias estimations even with a source. Additionaly, this shows that there is no drawback to use the one random point which is crucial in dimension three where the exact simulation is not possible.

\begin{tabular}{|c|cc|cr|}
\hline $\mathrm{h}$ & \multicolumn{2}{|c|}{ Exact simulation } & \multicolumn{2}{c|}{ One random point } \\
& Error & $\mathrm{CPU}$ & Error & $\mathrm{CPU}$ \\
\hline 0.2 & 0.017 & 210 & 0.016 & 176 \\
0.3 & 0.039 & 184 & 0.038 & 163 \\
0.4 & 0.07 & 173 & 0.069 & 144 \\
0.5 & 0.15 & 169 & 0.15 & 137 \\
\hline
\end{tabular}

Table 2: Parameters: $\left(x_{1}, x_{2}\right)=(0.5,0.5), N=10^{7}, \beta=0.1, \varepsilon=10^{-6}$

\subsubsection{Robin problem with damping}

We consider the equation

$\left\{\begin{array}{c}-\frac{1}{2} \Delta u+\lambda u=\lambda\left(\cos x_{1}-\sin x_{1}\right) e^{x_{2}}, x \in B_{1} \\ u+\beta \frac{\partial u}{\partial n}=-\beta x_{1} e^{x_{2}}\left(\sin x_{1}+\cos x_{1}\right)+e^{x_{2}}\left(\beta x_{2}+1\right)\left(\cos x_{1}-\sin x_{1}\right), x \in \partial B_{1}\end{array}\right.$

which exact solution is

$$
u\left(x_{1}, x_{2}\right)=\left(\cos x_{1}-\sin x_{1}\right) e^{x_{2}} .
$$

We compute with three different methods an approximate solution at point $\left(x_{1}, x_{2}\right)=(-0.7,-0.2)$ using $N=5.10^{6}$ simulations with $\beta=2, \lambda=0.1$. The values of $\beta$ and $\lambda$ are chosen so that the number of hits of the boundary is large and still close to a $O\left(\frac{1}{h}\right)$. These three methods are the WOS with a killing rate (KWOS) of section 3.3.2, the Standard Euler scheme and the Euler scheme 


\begin{tabular}{|c|cc|cc|rr|}
\hline $\mathrm{h}$ & \multicolumn{2}{|c|}{ KWOS } & \multicolumn{2}{c|}{ KEuler } & \multicolumn{2}{c|}{ Euler } \\
& Error & CPU & Error & CPU & Error & CPU \\
\hline 0.2 & 0.003 & 288 & 0.012 & 637 & 0.011 & 725 \\
0.3 & 0.0074 & 165 & 0.013 & 179 & 0.024 & 202 \\
0.4 & 0.016 & 104 & 0.033 & 76 & 0.04 & 85 \\
0.5 & 0.025 & 72 & 0.06 & 40 & 0.07 & 45 \\
0.6 & 0.035 & 51 & 0.09 & 26 & 0.1 & 28 \\
\hline
\end{tabular}

Table 3: Parameters: $\left(x_{1}, x_{2}\right)=(-0.7,-0.2), N=5.10^{6}, \beta=2, \lambda=0.1$

with a killing rate (KEuler) (section 3.3.1). Regardless the precomputation errors of the KWOS method, the global bias of this method is $O\left(\frac{1}{h}\left(\varepsilon+h^{3}\right)\right)$ where $\varepsilon$ is the absorption parameter of the WOS method. If we choose $\varepsilon=h^{3}$, we obtain a global order of a $O\left(h^{2}\right)$. For the two methods relying on the Euler scheme, the global bias is a $O\left(\frac{1}{h}\left(\Delta^{s}+h^{3}\right)\right)$ where $\Delta$ is the parameter of the Euler scheme. The parameter $s$ is unknown because our method combines the half-space approximation and the killing rate. We choose nevertheless $\Delta=h^{3}$ in order to compare with the KWOS method and because $s$ should be close to one as the killing rate is small. We observe that the KWOS method is the most efficient among the three methods. The approximation of its order is 2.3 while the other have an order close to 2 . The KEuler method is slightly more efficient than the Standard Euler method because it is faster. We can add that the KWOS and KEuler methods would be even more efficient when $\lambda$ is larger.

\subsection{Three dimensional transmission problem}

We consider the following equation

$$
\left\{\begin{array}{c}
-\frac{1}{2} \nabla(a(x) \nabla u(x))=0, x \in D \\
u\left(x_{1}, x_{2}, x_{3}\right)=\left\{\begin{array}{l}
\exp \left(\frac{x_{1}}{a_{1}}\right) \cos \left(x_{2}+x_{3}\right), x \in \partial D, x_{1} \geqslant 0 \\
\exp \left(\frac{x_{2}}{a_{2}}\right) \cos \left(x_{2}+x_{3}\right), x \in \partial D, x_{1} \leqslant 0
\end{array}\right.
\end{array}\right.
$$

in the domain $D=[-1,1]^{3}$. We perform $N=10^{7}$ simulations to compute the solution at point $(0.1,0,0)$ with the diffusion coefficients equal to $a_{1}=$ $1, a_{2}=0.5$. This point is chosen close to the center of the domain in order to increase the mean number of hits of the interface. This enables to discriminate more efficiently the three different methods that we will compare. The first one is the stochastic finite differences with parameter $h$ corresponding to the second choice in section (4.4.3). With probability $\frac{1}{3}$, the motion goes to $D_{2}$ and it goes to $D_{1}$ with probability $\frac{2}{3}$. The replacement points are chosen according to formula (4.17). For the second method, the probabilities to go to the two subdomains are the same but there is only two replacement points chosen normally at a distance $h$ to the interface [20]. The third method is the Kinetic approximation introduced in [15]. In this method, the motion goes equiprobably to $D_{1}$ and $D_{2}$ but it goes further inside the subdomain when the 
diffusion coefficient is larger. The replacement positions are not on a finite discrete grid but are continuous.

The exact solution of this equation is not known. The reference value $u(0.1,0,0) \simeq 0.9709$ has been obtained using the first method with parameter $h=0.01$ and a very large number of simulations $N=10^{8}$. In figure 2 , we plot in a logarithm scale the error at point $(0.1,0,0)$ as a function of CPU times. We can see that the stochastic finite differences (Diff2) is the most efficient followed by the kinetic approximation. The second method (Diff1) is clearly less efficient than the two previous ones. We can see that the order of the error compared to the CPU times is roughly equal to two for Diff2 and Kinetic methods and slightly less for the Diff1 method.

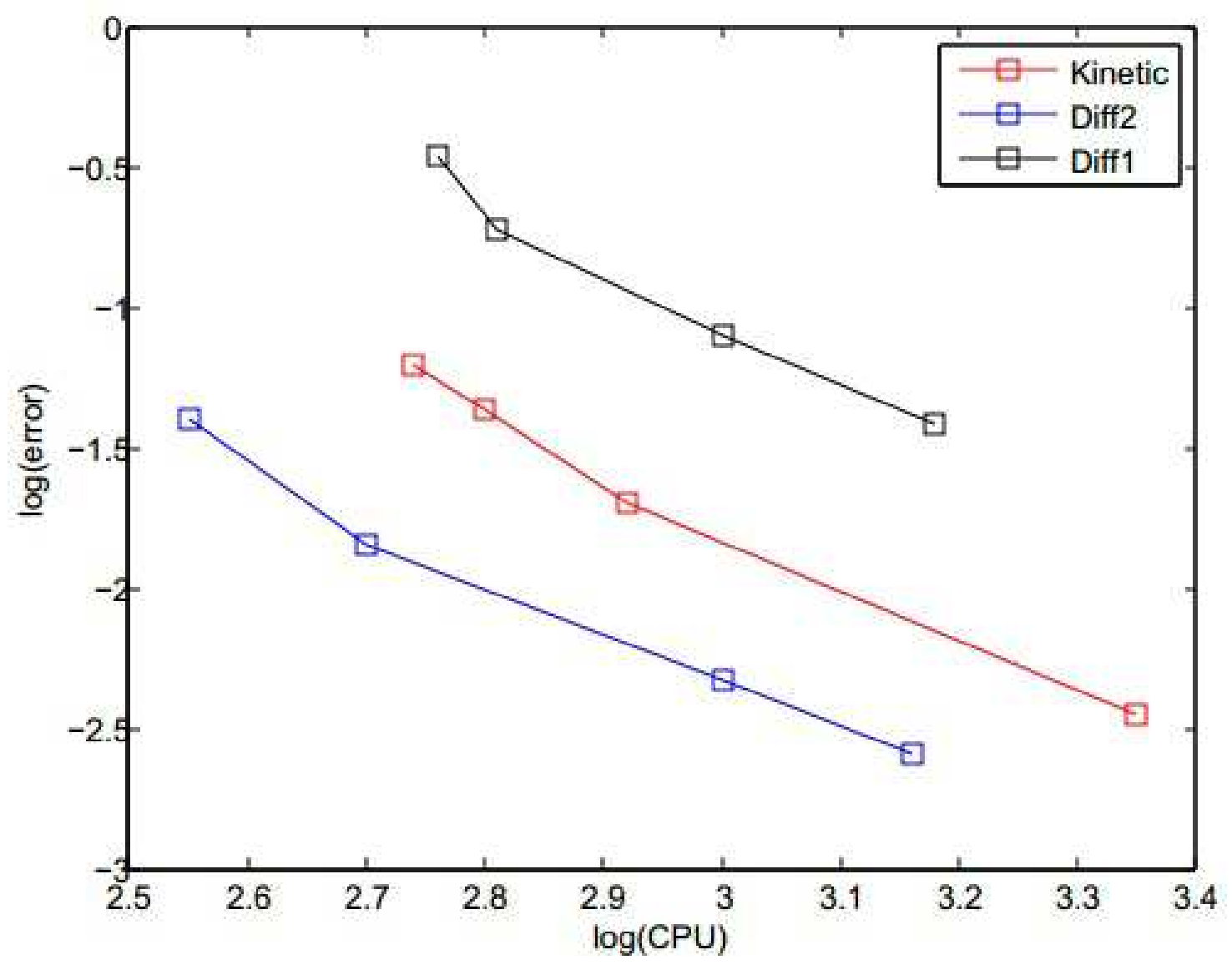

Figure 2: Comparison of error vs. CPU

\section{Conclusion}

In this paper, we have introduced new stochastic finite differents techniques to deal with boundary and interface conditions for general diffusion operator in stratified media in dimension two and three. These techniques induced a local bias of order three in Monte Carlo algorithms which leads generally to a global 
bias of order two. To deal with equations involving a damping term, we also need to modify the usual versions of the Euler schema and of the WOS method. Some numerical tests have confirmed our bias estimations and the efficiency of our new approach compared to standard ones. In future works, we intend to use these new tools for the forward resolution of inverse problems occuring for instance in electrical impedance tomography [10] or for the numerical solution of the linear or non-linear Poisson-Boltzmann equation [2]. 


\section{References}

[1] A. N. Borodin and P. Salminen, Handbook of Brownian motion facts and formulae, 2nd edition, Birkhauser, 2002.

[2] M. Bossy, N. Champagnat, S. Maire, and D. Talay, Probabilistic interpretation and random walk on spheres algorithms for the Poisson-Boltzmann equation in Molecular Dynamics, ESAIM M2AN 44 (2010), no. 5, 997-1048.

[3] M. Bossy, E. Gobet, and D. Talay, A symmetrized Euler scheme for an efficient approximation of reflected diffusions, J. Appl. Probab. 41 (2004), no. 3, 877-889.

[4] R.S. Cantrell and C. Cosner, Diffusion Models for Population Dynamics Incorporating Individual Behavior at Boundaries: Applications to Refuge Design, Theoretical Population Biology 55 (1999), no. 2, 189-207.

[5] M. Deaconu and A. Lejay, A random walk on rectangles algorithm, Methodol. Comput. Appl. Probab. 8 (2006), no. 1, 135-151.

[6] R. Erban and S. J. Chapman, Reactive boundary conditions for stochastic simulations of reaction diffusion processes, Phys. Biol. 4 (2007), no. 1, 16-28.

[7] P. Etoré, On random walk simulation of one-dimensional diffusion processes with discontinuous coefficients, Electron. J. Probab. 11 (2006), no. 9, 249-275.

[8] E. Gobet, Euler schemes and half-space approximations for the simulation of diffusion in a domain, ESAIM Probability and Statistics, 5 (2001), 261-297.

[9] D. S. Grebenkov, Subdiffusion in a bounded domain with a partially absorbing-reflecting boundary, Physical review E 81, 021128, 2010.

[10] M. Hanke, M. Bruhl, Recent progress in electrical impedance tomography, Inverse Problems, 19 (2003), 1-26.

[11] C.O. Hwang, M. Mascagni, and J.A. Given, A Feynman-Kac path-integral implementation for Poisson's equation using an $h$ conditioned Green's function. Math. Comput. Simulation, 62 (2003) no. 3-6, 347-355.

[12] H. Hoteit, R. Mose, A. Younes, F. Lehmann, and Ph. Ackerer, Three-dimensional modeling of mass transfer in porous media using the mixed hybrid finite elements and the random-walk methods, Math. Geology 34 (2002), no. 4, 435-456. 
[13] B. Lapeyre, E. Pardoux and R. Sentis, Méthodes de MonteCarlo pour les équations de transport et de diffusion. Collection Mathématiques et Applications 29 - Springer Verlag, 1998.

[14] A. Lejay, Simulation of a stochastic process in a discontinuous layered medium, Electron. Comm. Probab. 16 (2011), 764-774.

[15] A. Lejay and S. Maire, Simulating diffusions with piecewise constant coefficients using a kinetic approximation, Comput. Methods Appl. Mech. Engrg. 199 (2010), no. 29-32, 2014-2023.

[16] A. Lejay and S. Maire, New Monte Carlo schemes for simulating diffusions in discontinuous media, Journal of Computational and Applied Mathematics, 245 (2013), 97-116.

[17] S. Maire and E. Tanré, Monte Carlo approximations of the Neumann problem (2012). Preprint.

[18] S. Maire and E. Tanré, Some new simulation schemes for the evaluation of Feynman-Kac representations, Monte Carlo methods Appl. 14 (2008), no. 1, 29-51.

[19] M. Martinez, Interprétations probabilistes d'opérateurs sous forme divergence et analyse de méthodes numériques associées, Ph.D. thesis, Université de Provence / INRIA Sophia- Antipolis, 2004.

[20] M. Mascagni and N. A. Simonov, Monte Carlo methods for calculating some physical properties of large molecules, SIAM J. Sci. Comput. 26 (2004), no. 1, 339-357.

[21] G.A. Mikhallov and R.N. Makarov, Solution of boundary value problems of the second and third kind by the Monte Carlo methods, Siberian Math. J. 38 (1997), no. 3, 518-527.

[22] G. N. Milstein, M. V. Tretyakov, Stochastic numerics for mathematical physics, Springer-Verlag, Berlin, 2004.

[23] M. E. Muller, Some continuous Monte Carlo methods for the Dirichlet problem, Ann. Math. Statist. 27 (1956), 569-589.

[24] K. K. Sabelfeld, Monte Carlo methods in boundary value problems, Springer Series in Computational Physics, Springer-Verlag, Berlin, 1991. Translated from the Russian.

[25] N. A. Simonov, Walk-on-spheres algorithm for solving boundaryvalue problems with continuity flux conditions. Monte Carlo and quasi-Monte Carlo methods 2006, 633-643, Springer, Berlin, 2008 . 
[26] D. W. Stroock, Diffusion semigroups corresponding to uniformly elliptic divergence form operators, Séminaire de Probabilités, XXII, Lecture Notes in Math., vol. 1321, Springer, Berlin, 1988, $316-347$.

[27] D. Talay, L. Tubaro Expansions of the global error for numerical schemes solving stochastic differential equations, Stochastic Analysis and Applications, 8, no.4 (1990), 94-120.

[28] E.C. Zachmanoglou, D.W. Thoe, Introduction to Partial Differential Equations with Applications, Dover, New York, 1976.

[29] M. Zhang, Calculation of diffusive shock acceleration of charged particles by skew Brownian motion, Astrophys. J. 541 (2000), 428-435. 